\title{
Dizziness in A Patient with Airway Compromise and TMD: A Case Report
}

\author{
Charles Blum* and Jeffrey A Mersky \\ Director of Research, Sacro Occipital Technique Organization-USA, Ocean Park Boulevard, Santa Monica, California, USA
}

*Corresponding author: Charles Blum, Director of Research, Sacro Occipital Technique Organization-USA, Ocean Park Boulevard, Santa Monica, California, USA
Received Date: February 28, 2020

Published Date: March 20, 2020

\section{Introduction}

Evidence is emerging to support the theory that airway oxygenation, which is often associated with obstructive sleep apnea (OSA), is an important condition to include in a differential diagnosis. OSA is a condition that sometimes presents as a symptom of a more complex condition (e.g., cardiovascular, diabetes, cancer, inflammatory diseases, gastroesophageal reflux disorder, etc.) [1-10] without an apparent direct relationship to any airway compromise. One feature of OSA is the jaw-head position-hypoxia relationship that is associated with a persistent forward head posture [11-14] causing chronic skeletal pains that do not resolve with conventional therapies. As a means to sustain optimal airway space with OSA the head tends to move forward and while this improves oxygenation it adversely affects the cervical spine and body posture. Literature suggests there is a relationship between head posture and temporomandibular joint dysfunction (TMD) issues [15-20].

\section{Case History}

A 38-year-old patient presented for care at this office in November 2016 with an array of symptoms (autoimmune disorders, dizziness, obstructive sleep apnea-OSA, etc.). The onset of his condition reportedly began eight years ago following a surfing accident (fell on left shoulder) and removal of his wisdom teeth. After these events, he began experiencing syncope, brain fog, dizziness, and movement/vertigo, all of which worsen when sitting on soft seats or when hunching over or with his head tilted up or down. He noted that his condition was aggravated with computer work and when his head was in a forward posture. Brain fog prevented him from being able to concentrate at work, and he found his condition debilitating, relentless, and life-altering. He was primarily diagnosed with apnea (OSA), vestibular dysfunction, vascular headaches/migraines, and cervico/thoracic enthesopathy.

At eight years old, the patient reports that he fell off of a cliff and landed on his head. At age 10, he fell onto a skill saw blade and needed multiple stitches to the back of his head. The patient had an automobile accident at 14 years old, which resulted in head trauma and a fractured $\mathrm{C} 7$ spinous process. He had his adenoid and tonsils removed at the age of 21 . Compounding his presentation, the patient had migraines and sleep disorders which were diagnosed as a form of sleep apnea in 2008. He has had a history of anemia with a low red blood cell count complicating his hypoxic condition. Of note he was temporarily disabled for 2 months in 2008 and during that time received chiropractic care over the course of a month. The patient reported that chiropractic helped him somewhat but did not affect the dizziness or brain fog. He was also on disability for 1 month in February 2017 due to his condition.

A 2010 ECG study showed probable left ventricular hypertrophy of heart, and ejection fraction at $67 \%$ of normal. EEG testing at that time showed some brief high voltage bursts of alpha activity followed by some decrease in the background activity. A recent MRI of the brain and head was described as normal. He was recently diagnosed with scleroderma but was not receiving care for that condition at the time of his presentation at this office. Since early 2012 he was using a continuous positive airway pressure (CPAP) machine for sleep and as a means to treat his OSA [21].

The patient had been to 28 different healthcare practitioners including several Chiropractors (utilizing upper cervical technique, sacro occipital technique, diversified technique, and nasal balloon technique, etc.), medical doctors (cardiologist, neurologist, ear 
nose and throat specialist, allergy-immunologist, sleep specialist, internist, rheumatologist), cranial osteopaths, acupuncturists, physical therapists, and massage therapists. He has spent thousands of dollars on care and had been on short-term disability for the past year. For three years prior to beginning care at this office he had been unable to perform physical exercise. With regards to the other care he received, he reported that he felt somewhat better, though only temporarily, with upper cervical chiropractic treatment.

\section{Methods/Interventions}

The patient's examination findings revealed reduced cervical rotation, and pain with lateral cervical flexion localizing to the occiput /C1 and C5 through C7. Lumbar vertebral flexion was limited with pain localizing to the L5 region. Sacro Occipital technique (SOT) cervical compaction test was positive for lumbopelvic involvement. Cervical stairstep was positive at the Occiput /C1 and C5-7 spinal levels. Forward head posture was noted with sensitivity on palpation at the right temporal bone styloid process (can be related to L5 [22,23] and the right 1st costotransverse junction. Body sway analysis noted both anterior/posterior and lateral sway patterns. He had sleep disorders/apnea with inability to reach deep sleep, stage 3-4, associated with frequent limb movements at the time he should enter deep sleep treated with a dental appliance and CPAP.

Treatment focused on SOT and SOT cranial chiropractic interventions, nasal balloon methods for craniofacial sinus expansion, and supplementation with B12/methylated folic acid (to facilitate red blood cell oxygen uptake) [24]. SOT interventions focused on releasing right psoas and bilateral piriformis muscles' greater trochanteric attachments. The patient was treated for SB+ pattern, which relates to increased lumbosacral dural meningeal tensions [25]. His cervical spine was treated with cervical stairstep mobilization and adjusting. Osseous adjusting was applied to the thoracic and lumbar vertebra along with an anterior diaphragm pseudo-hiatal hernia release. Common cranial patterns included sphenobasilar right side-bending and vertical strain lesions [26]. Intraoral adjusting focused on releasing his right zygoma that was restricted in external rotation and bilaterally external pterygoid muscle release. The left sphenoidal greater wing was also corrected from an external rotation positioning. Initial use of nasal balloons focused on insertion into the lower right and middle left turbinates, followed with upper and middle turbinate releases [27] on alternate days.

\section{Results}

The patient was treated from November 15, 2016 through March 24, 2017. By early 2017 his eight-year bout with dizziness was reduced and he could function in most activities of daily living. By March 2017 he only noted mild neck pain (50\% reduction) with significantly reduced tension and swelling. His lower back pain would only present on occasion and described by the patient to be $80 \%$ improved. Since he was using the CPAP and an oral dental appliance, he noted an improvement in his oxygenation characterized by a pulse oximeter reading of $98 \%$, and a normal Epworth Sleepiness Scale scoring [28]. Initial pre-treatment (November 2016) Roland-Morris disability index scales [29] were scored at $28 \%$ for low back pain and disability and with a followup approximately six months later showing a minimal change or reduction to $24 \%$, a reduction of $4 \%$. His neck pain disability index scales [30] were initially measured at $52 \%$, with follow-up testing (April 2017) showing a reduction of his neck disability index of $36 \%$, a reduction of $16 \%$. Beck's motor testing [31-34] was normal for TMJ dysfunction and positive for his neck at the initial office visit and reported as normal after 2 nd office visit.

The patient reported that the chiropractic care he received at this office, which consisted of sacro occipital technique (SOT) along with SOT extra cranial/intra oral and nasal balloons gave him at least $50 \%$ improvement of his symptoms, which had been unresponsive for eight years, along with giving him the ability to return to work. After approximately five months of care the patient has been able to maintain his improvements and continues to feel increasingly capable of doing his activities of daily living without restrictions. He still self-limits, particularly regarding heavy strenuous work, though for the first time in years he has been able to do rock climbing and other limited sports activities.

\section{Discussion}

The care for this patient's complex presentation was multifactorial with a focus on:

1. Improving airway and oxygenation.

2. Balancing pelvic imbalances affecting and being affected by his compromised visual and vestibular righting.

3. Balancing cranial and craniofacial imbalances, possibly affected by the multiple head traumas and a secondary response to wisdom teeth extraction.

4. Consideration of inflammatory and autoimmune compromise.

Improving airway and oxygenation: The patient had been suffering from OSA and was being treated with a CPAP and nighttime dental appliance. However, one consideration is what would be happening during the day when the patient was not using a CPAP or dental appliance to enhance his airway function. While he did have tonsil and adenoid surgery, further improvement to his airway space may have occurred using the nasal balloon techniques to reduce any turbinate restriction and expand some nasopharyngeal pathway space. One consideration for care was the mechanical expansion of the craniofacial airway in order to improve oxygen intake, and another aspect involved improving red blood cell production by utilizing vitamins B12 and a methylated folic acid supplement [35,36].

Balancing pelvic imbalances affecting and being affected by his compromised visual and vestibular righting: Many of the factors 
associated with posture balancing and visual and vestibular righting focus upon assessing the patient in a standing position [37]. This patient's initial presentation was quite unusual because he could function with less dizziness when standing; however, if he sat upon a soft or cushiony surface, he would become dizzy. This suggested that stressors from pelvic or lumbopelvic imbalance were having an effect on his visual and vestibular righting centers, and that these centers may have already been compromised from the prior head traumas [38].

Balancing cranial and craniofacial imbalances possibly affected by the multiple head traumas and secondary response to wisdom teeth extraction: While it is possible that the pelvic imbalance had a negative effect on the patient's visual and vestibular righting leading to chronic dizziness, it is also likely that the multiple head traumas led to his compromised ability to handle this increased stress $[39,40]$ SOT and SOT cranial techniques such as those used in this study have been used for the treatment of head trauma and post-concussion syndromes [41-44]. Patients treated with SOT and SOT cranial techniques have presented with minor head trauma with subsequent headaches, dizziness and limited function[41] significant head trauma(s) associated with concussions leading to various conditions such as, OSA, TMJ disorders, and inability to function or perform their activities of daily living [42-44].

Consideration of inflammatory and autoimmune compromise: Due to the complex and chronic nature of the patient's presentation, genetic testing will need to be performed to help determine if he does have the methylenetetrahydrofolate reductase (MTHFR) gene or any possible genetic defects [45] affecting his health condition. Since inflammatory issues can be related to the lack of having the MTHFR gene it may be helpful to assess if he has a genetic pre-disposition to scleroderma [46]. As a means to facilitate reduction of any inflammatory contribution to his condition dietary recommendations were given to him as of April 2017 for an antiinflammatory diet $[47,48]$ and food sensitivity tests are also being scheduled.

This case is interesting as the patient had been to so many health practitioners without much help yet responded most notably and with lasting results when receiving SOT chiropractic care. As with any case study there are limitations to generalizing its findings to the population at large. This is because this report is only a single subject case study that didn't include controls, sham grouping, and randomization. In addition, various possibilities for the positive outcomes, including placebo, ideomotor, regression to the mean, and others cannot be ruled out. Yet the temporal relationship between the care rendered and the prior unresponsiveness to other care makes this case and its results compelling.

\section{Conclusion}

This is a complex case report of a 38-year-old patient seen for care at this office for various symptoms that included autoimmune disorders, dizziness, OSA, TMD, migraines and skeletal pain patient. His condition had not been stable for eight years prior to the specific care rendered, which included SOT, SOT cranial, nasal balloon, and nutritional modification. It was difficult to directly assess what aspects of his presentation were genetic, macrotrauma-induced, or the result from microtraumas, though the care rendered appeared effective regardless. Further research is needed to determine which subsets of patients with these types of complex conditions might also respond to the type of care rendered this patient.

\section{Acknowledgement}

None.

\section{Conflict of Interest}

The authors declare no conflict of interests.

\section{References}

1. Gozal D, Farré R, Nieto FJ (2016) Obstructive sleep apnea and cancer: Epidemiologic links and theoretical biological constructs. Sleep Med Rev 27: 43-55.

2. Gilat H, Vinker S, Buda I, Soudry E, Shani M, et al. (2014) Obstructive sleep apnea and cardiovascular comorbidities: a large epidemiologic study. Medicine (Baltimore) 93(9): e45.

3. Russell MB, Kristiansen HA, Kværner KJ (2014) Headache in sleep apnea syndrome: epidemiology and pathophysiology. Cephalalgia 34(10): 752755.

4. Hirotsu C, Tufik S, Guindalini C, Mazzotti DR, Bittencourt LR (2013) Association between uric acid levels and obstructive sleep apnea syndrome in a large epidemiological sample. PLoS One 8(6): e66891.

5. Emilsson OI, Janson C, Benediktsdóttir B, Júlíusson S, Gíslason T (2012) Nocturnal gastroesophageal reflux, lung function and symptoms of obstructive sleep apnea: Results from an epidemiological survey. Respir Med 106(3): 459-466.

6. Lurie A (2011) Obstructive sleep apnea in adults: epidemiology, clinical presentation, and treatment options. Adv Cardiol 46: 1-42.

7. Madani M, Madani F (2011) Epidemiology, pathophysiology, and clinical features of obstructive sleep apnea. Oral Maxillofac Surg Clin North Am 21(4): 369-375.

8. Hernández C, Durán-Cantolla J, Lloberes P, González M (2009) Innovations in the epidemiology, natural history, diagnosis and treatment of sleep apnea-hypopnea syndrome. Arch Bronconeumol 45(1): 3-10.

9. Mekhitarian Neto L, Fava AS, Lopes HC, Stamm A (2005) Epidemiological analysis of structural alterations of the nasal cavity associated with obstructive sleep apnea syndrome (OSA). Braz J Otorhinolaryngol 71(4): 464-466.

10. Young T, Peppard PE, Gottlieb DJ (2002) Epidemiology of obstructive sleep apnea: a population health perspective. Am J Respir Crit Care Med 165(9): 1217-1239.

11. Inoko Y, Morita O (2009) Influence of oral appliances on craniocervical posture in obstructive sleep apnea-hypopnea syndrome patients. J Prosthodont Res 53(3):107-10.

12. Tong M, Sakakibara H, Xia X, Suetsugu S (2000) Compensatory head posture changes in patients with obstructive sleep apnea. J Tongji Med Univ 20(1): 66-69.

13. Tangugsorn V, Krogstad O, Espeland L, Lyberg T (2000) Obstructive sleep apnea (OSA): a cephalometric analysis of severe and non-severe OSA patients. Part I: Multiple comparison of cephalometric variables. Int J Adult Orthodon Orthognath Surg 15(2):139-52. 
14. Bacon W, Berreur C, Krieger J, Hildwein M, Stierle JL (1992) Pharyngeal obstruction and the functional adaptation of the natural posture of the head and the hyoid bone in sleep apnea syndrome. Orthod Fr 63(2): 595-602.

15. Fuentes Fernández R, Carter P, Muñoz S, Silva H, Oporto Venegas GH, et al. (2016) Evaluation of validity and reliability of a methodology for measuring human postural attitude and its relation to temporomandibular joint disorders. Singapore Med J 57(4): 204-208.

16. Ohmure H, Miyawaki S, Nagata J, Ikeda K, Yamasaki K, et al. A (2008) Influence of forward head posture on condylar position. J Oral Rehabil 35(11): 795-800.

17. Olmos SR, Kritz-Silverstein D, Halligan W, Silverstein ST (2005) The effect of condyle fossa relationships on head posture. Cranio 23(1): 4852 .

18. Gonzalez HE, Manns A (1996) Forward head posture: its structural and functional influence on the stomatognathic system, a conceptual study. Cranio 14(1): 71-80.

19. Lee WY, Okeson JP, Lindroth J (1995) The relationship between forward head posture and temporomandibular disorders. J Orofac Pain 9(2): 161-167.

20. Braun BL (1991) Postural differences between asymptomatic men and women and craniofacial pain patients. Arch Phys Med Rehabil 72(9): 653-656.

21. Ward K, Hoare KJ, Gott M (2014) What is known about the experiences of using CPAP for OSA from the users' perspective? A systematic integrative literature review. Sleep Medicine 18(4): 357-366.

22. Blum CL (2015) R+C Factors and Sacro Occipital Technique Orthopedic Blocking: a pilot study using pre and post VAS assessment. The Journal of the Canadian Chiropractic Association 59(2): 134-142.

23. Shaneyfelt D, Blum CL, Benner CD (2013) Styloid process sensitivity in a patient with low back pain and radicular syndrome: A case report. World Congress on Low Back \& Pelvic Pain Conference, Dubai.

24. Huang Y, Khartulyari S, Morales ME (2008) Quantification of key red blood cell folates from subjects with defined MTHFR $677 \mathrm{C}>\mathrm{T}$ genotypes using stable isotope dilution liquid chromatography/mass spectrometry. Rapid communications in mass spectrometry 22(16): 2403-2412.

25. Farmer JA, Blum CL (2002) Dural port therapy. J Chiropr Med 1(2): 5461.

26. Alma KD, Degenhardt BF, Snider KT, Johnson JC, Flaim MS, et al. (2008) Intraobserver reliability of cranial strain patterns as evaluated by osteopathic physicians: a pilot study. J Am Osteopath Assoc 108(9): 493502.

27. Berman S (1991) Skull Dysfunction. Cranio 9(3): 268-279.

28. Buysse DJ, Hall ML, Strollo PJ (2008) Relationships Between the Pittsburgh Sleep Quality Index (PSQI), Epworth Sleepiness Scale (ESS), and Clinical/Polysomnographic Measures in a Community Sample. J Clin Sleep Med 4(6): 563-571.

29. Stratford PW, Riddle DL (2016) A Roland Morris Disability Questionnaire Target Value to Distinguish between Functional and Dysfunctional States in People with Low Back Pain. Physiother Can 68(1): 29-35.

30. Jorritsma W, Dijkstra PU, De Vries GE, Geertzen JHB, Reneman MF (2012) Detecting relevant changes and responsiveness of Neck Pain and Disability Scale and Neck Disability Index. European Spine J 21(12): 2550-2557.
31. Beck JL (2008) Neurobiological basis for chronic pain. Practical Pain Management 8(6): 44-53.

32. Beck JL (2008) Practical application of neuropostural evaluations the PANE Process: basic principles and the first three tests. Practical Pain Management 8(7): 47-53.

33. Beck JL (2008) Neurodevelopmental basis for chronic regional pain syndrome. Practical Pain Management 8(9): 19-27.

34. Beck JL (2009) Application of spinal segmental physiology to evaluating chronic pain. Practical Pain Management 9(4): 12-19.

35. Koury MJ, Ponka P (2004) New insights into erythropoiesis: the roles of folate, vitamin B12, and iron. Annu Rev Nutr 24: 105-131.

36. Scott JM (1999) Folate and vitamin B12. Proc Nutr Soc 58(2): 441-418.

37. Morningstar MW, Pettibon BR, Schlappi H, Schlappi M, Ireland TV (2005) Reflex control of the spine and posture: a review of the literature from a chiropractic perspective. Chiropr Osteopat 13: 1-16.

38. Lovett L, Blum CL (2006) Behavioral and Learning Changes Secondary to Chiropractic Care to Reduce Subluxations in a Child with Attention Deficit Hyperactivity Disorder: A Case Study. Journal of Vertebral Subluxation Research 1-6.

39. Suarez H, Alonso R, Arocena M, Suarez A, Geisinger D (2012) Clinical characteristics of positional vertigo after mild head trauma. Acta Otolaryngol 131(4): 377-381.

40. Dalby BJ (1993) Chiropractic diagnosis and treatment of closed head trauma. J Manipulative Physiol Ther 16(6):392-400.

41. Blum CL (2002) Chiropractic Treatment of Mild Head Trauma: A Case History, Proceedings of the 2002 International Conference on Spinal Manipulation, Toronto Ontario, Canada.

42. Holbeck M (2000) Chiropractic treatment of a post-concussion syndrome secondary to volleyball injury in a 14-year-old female: A case report. J Chirop Ed 31(1): 1-64.

43. Bloink T, Blum CL (2015) Post-concussion syndrome, temporomandibular joint disorders, and chiropractic dental co-treatment: A case report. World Federation of Chiropractic's $13^{\text {th }}$ Biennial Congress/2015, Annual European Chiropractic Union, Athens, Greece.

44. Gerardo RC, Blum CL (2016) Sacro occipital technique and stomatognathic interventions in the treatment of a professional football player with multiple concussions, TBI, TMD, and OSA: A case report. 8th Annual Sacro Occipital Technique Research Conference: New Orleans, Louisiana.

45. Huang Y, Khartulyari S, Morales ME (2008) Quantification of key red blood cell folates from subjects with defined MTHFR 677C >T genotypes using stable isotope dilution liquid chromatography/mass spectrometry. Rapid commun Mass spectrum 22(16): 2403-2412.

46. Luderschmidt C, Scholz S, Mehlhaff E, König G, Albert E (1987) Association of progressive systemic scleroderma to several HLA-B and HLA-DR alleles. Arch Dermatol 123(9): 1188-1191.

47. Seaman DR (2002) The diet-induced proinflammatory state: a cause of chronic pain and other degenerative diseases? J Manipulative Physiol Ther 25(3): 168-179.

48. Fenech M (2001) The role of folic acid and Vitamin B12 in genomic stability of human cells. Mutat Res 475(1-2): 57-67. 